Supporting Information

\title{
Benchmarking the Orbitrap Tribrid Eclipse for Next Generation Multiplexed Proteomics.
}

\author{
AUTHORS \\ Devin K Schweppe ${ }^{1 *}$ \\ AUTHOR ADDRESS \\ 1 - Harvard Medical School, Boston, MA 02115 \\ 2 - Thermo Fisher Scientific, San Jose, CA 95134 \\ * - Correspondence should be addressed to: devin_schweppe@hms.harvard.edu
}

Qing Yu ${ }^{1}$, Joao A Paulo ${ }^{1}$, Jose Naverrete-Perea ${ }^{1}$, Graeme C McAlister ${ }^{2}$, Jesse D Canterbury ${ }^{2}$, Derek J Bailey ${ }^{2}$, Aaron M Robitaille ${ }^{2}$, Romain Huguet ${ }^{2}$, Vlad Zabrouskov ${ }^{2}$, Steven P Gygi ${ }^{1}$, 


\section{Supporting Information Contents}

\section{Figures}

Figure S1 - Characterization of multiplexed quantification based on TurboTMT, isolation specificity, and instrument peak shape.

Figure S2 - TKO standard analysis for Ura2 and His4 peptides.

Figure S3 - Heatmap and PCA of 11 cell line sample.

Figure S4 - Number and percentage of PSMs that are removed due to each filtering criteria.

Figure S5 - Quantitative comparison on additional cell lines.

Figure S6 - Total MCF7 proteins quantified with a fold change greater than two-fold compared to the mean of all cell lines.

\section{Tables}

Table S1 - Summary of normalized isotope ratio for quadrupole isolation test ( $\mathrm{z}=2$ and 3 ), related to Figure 1D.

Table S2 - Summary of normalized summed intensity for quadrupole isolation test with offset ( $\mathrm{z}=2$ and 3 ), related to Figure 1F. 

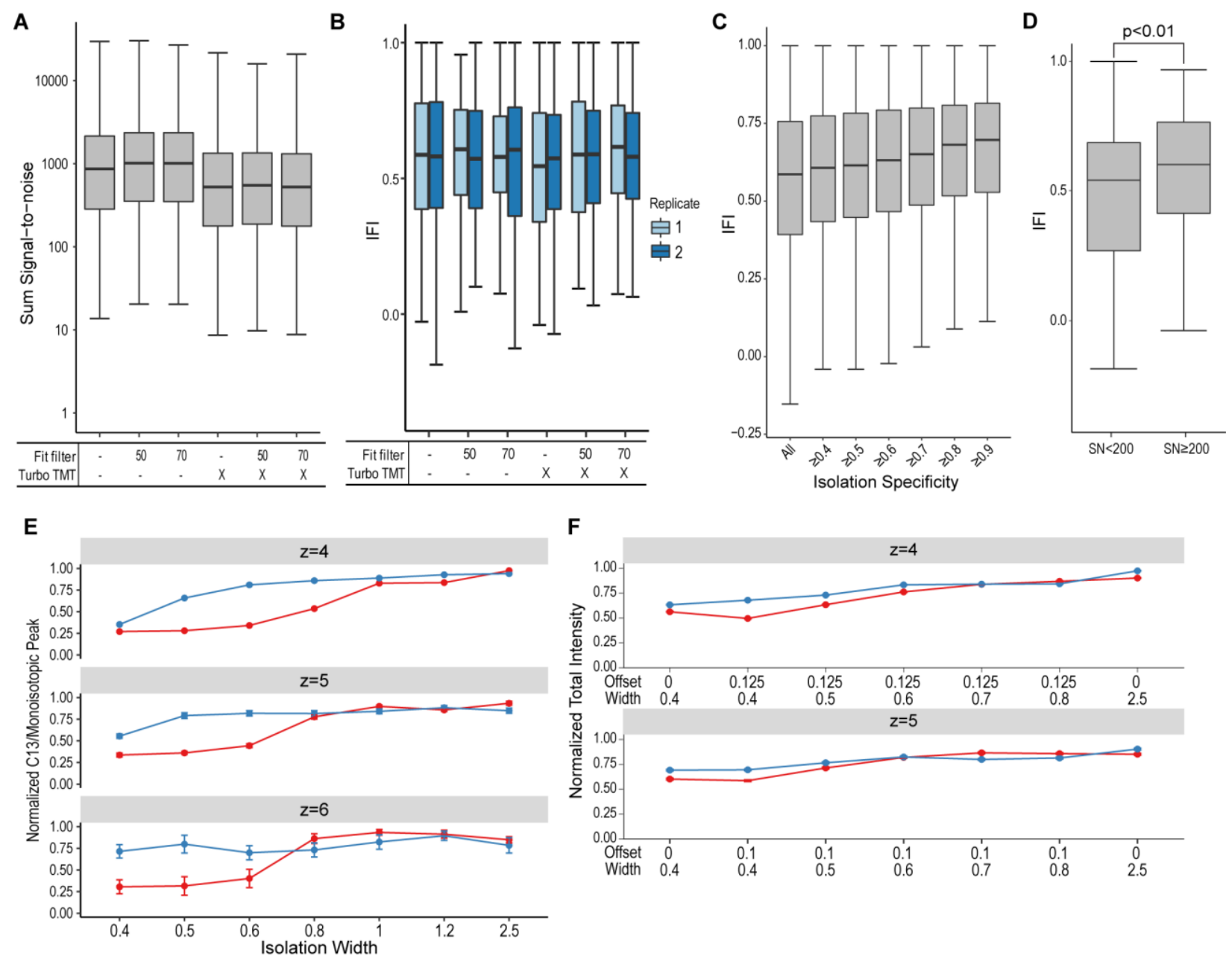

Figure S1. (A) Summed signal-to-noise (SN) distribution for each method tested. (B) Interference free index (IFI) for each method tested. (C) IFI versus isolation specificity. IFI can be improved with increasing isolation specificity. (D) IFI values for PSMs with summed SN $<200$ were statistically lower than those with summed $\mathrm{SN} \geq 200$. (E) Ratios between ${ }^{13} \mathrm{C}$ and monoisotopic peaks (Mean $\pm \mathrm{SEM}$ ), normalized to the maximal value observed for charge states 4, 5 and 6. (F) Summed signal of monoisotopic and ${ }^{13} \mathrm{C}$ peaks with various isolation width and offset settings for charge states 4 and 5 , normalized to the maximal value observed. 

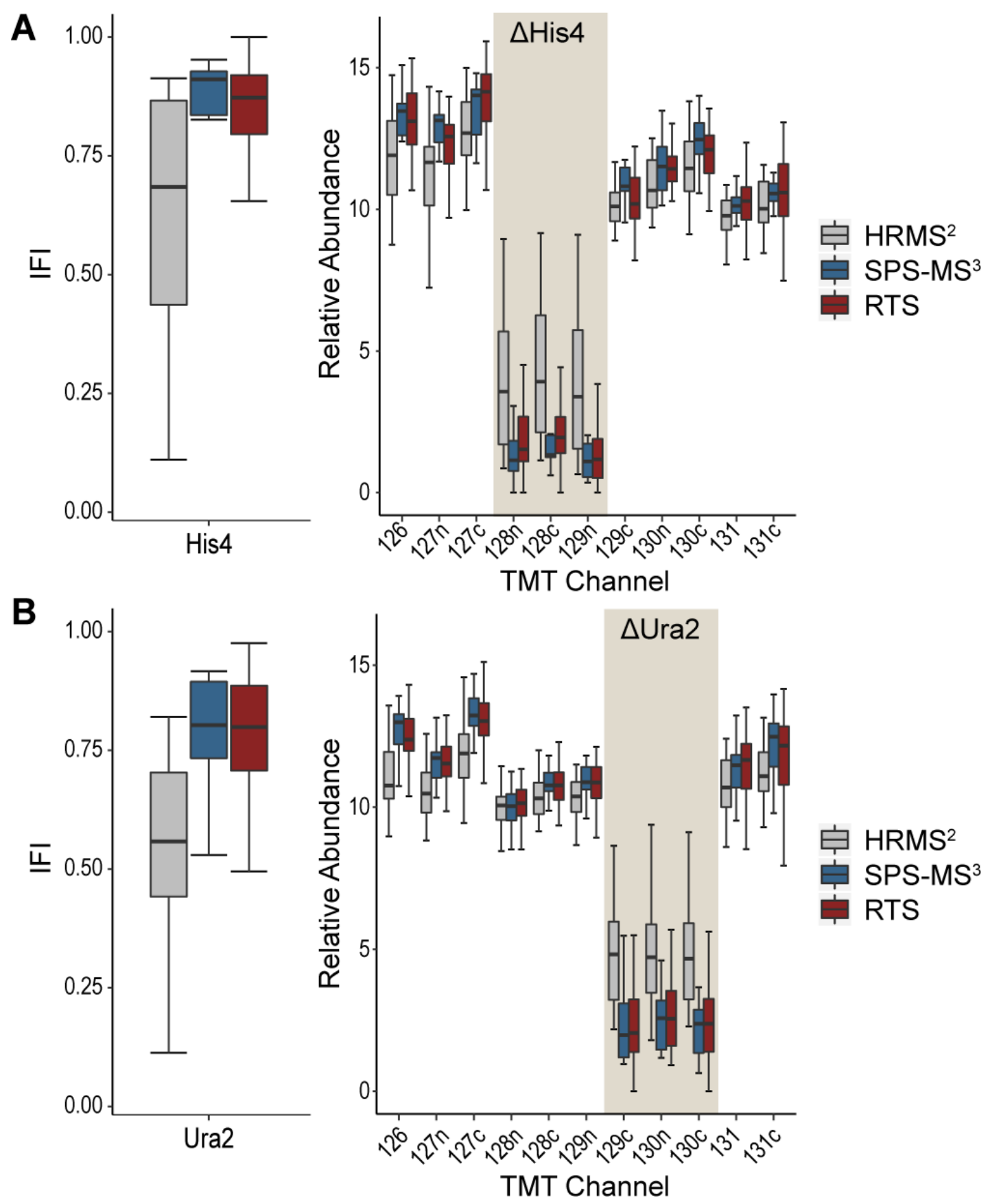

Figure S2. (A) IFI of His4 peptides (left) and relative abundance of contributing peptides in each TMT channel (right). (B) IFI of Ura2 peptides (left) and relative abundance of contributing peptides in each TMT channel (right). 
A

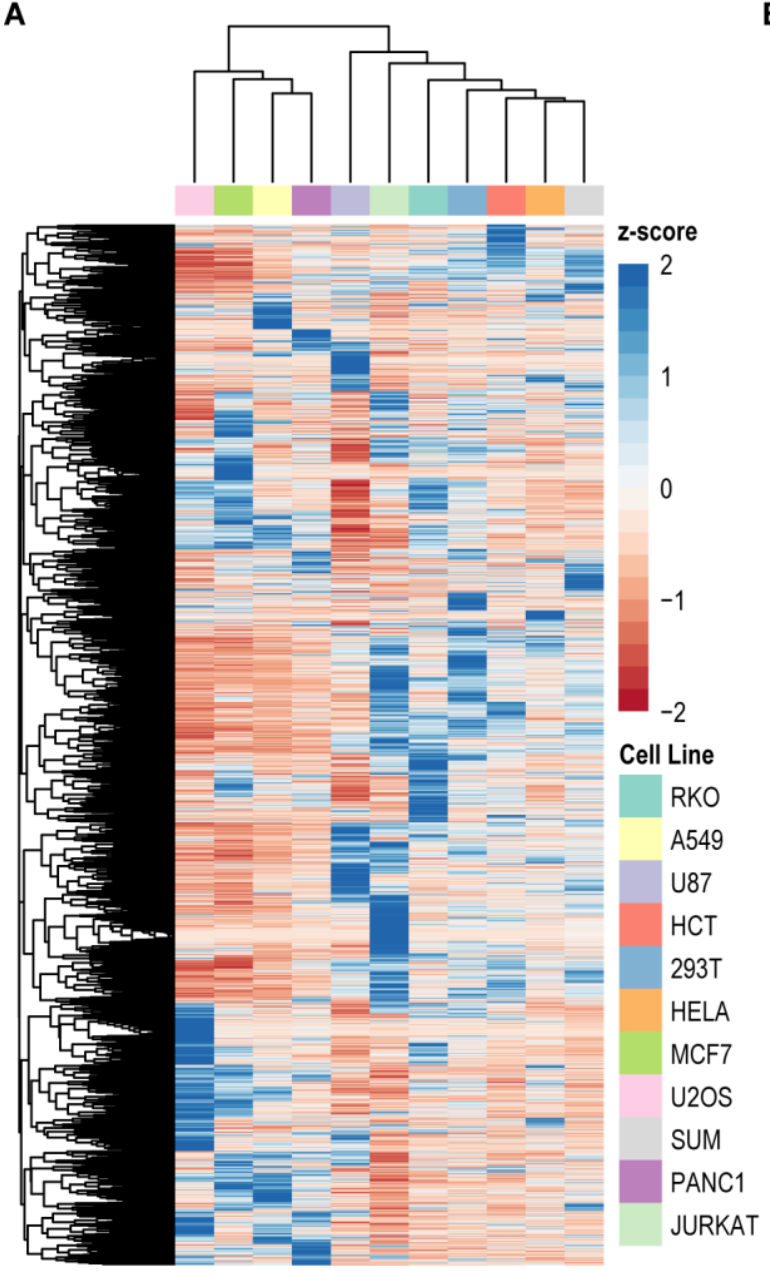

B

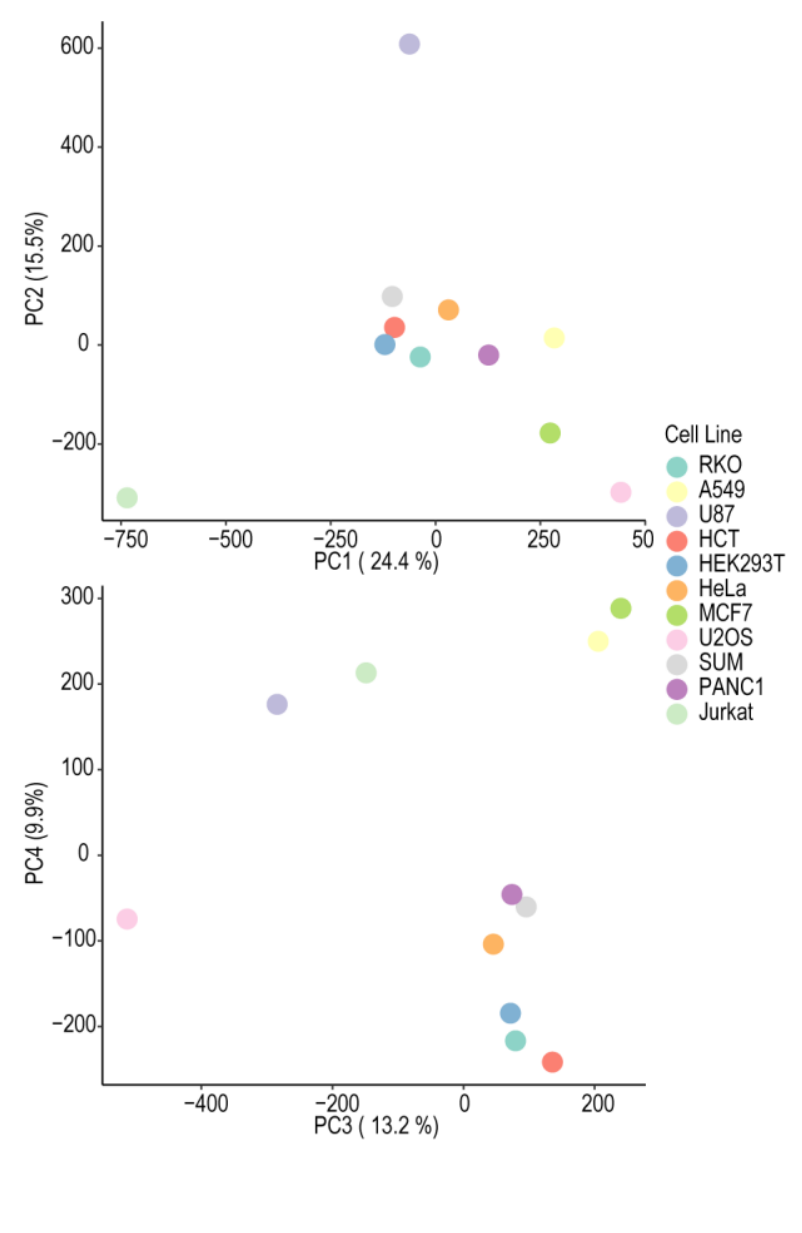

Figure S3. Heatmap and PCA of 11 cell line sample. (A) Heat map and hierarchical clustering of eleven cell line proteomics using data collected with RTS. (B) Principal component analysis results using the same data set. 

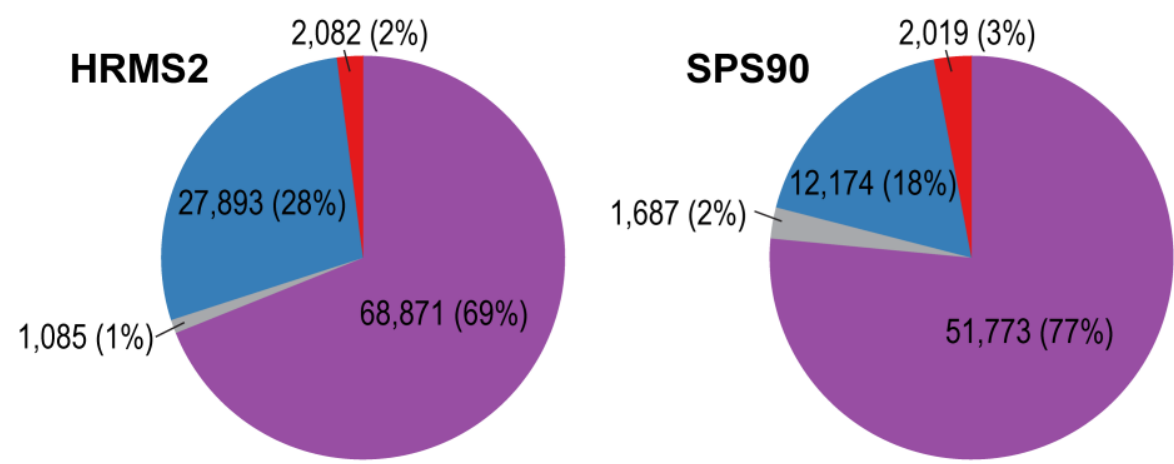

\section{Peptide filtering}
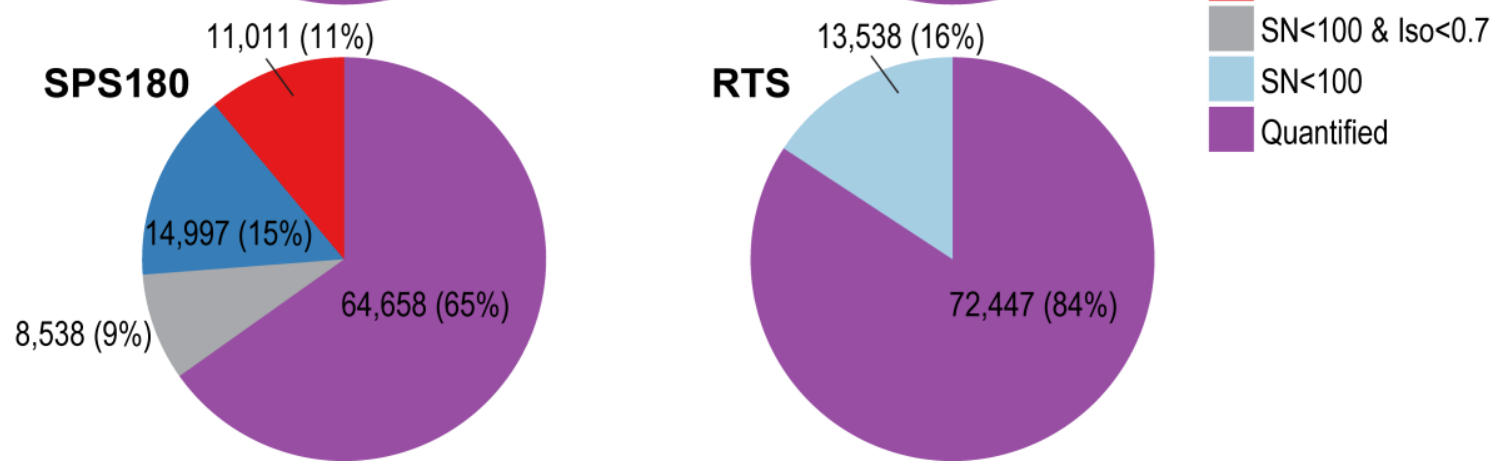

Figure S4. Number and percentage of PSMs that are removed due to each filtering criteria. 

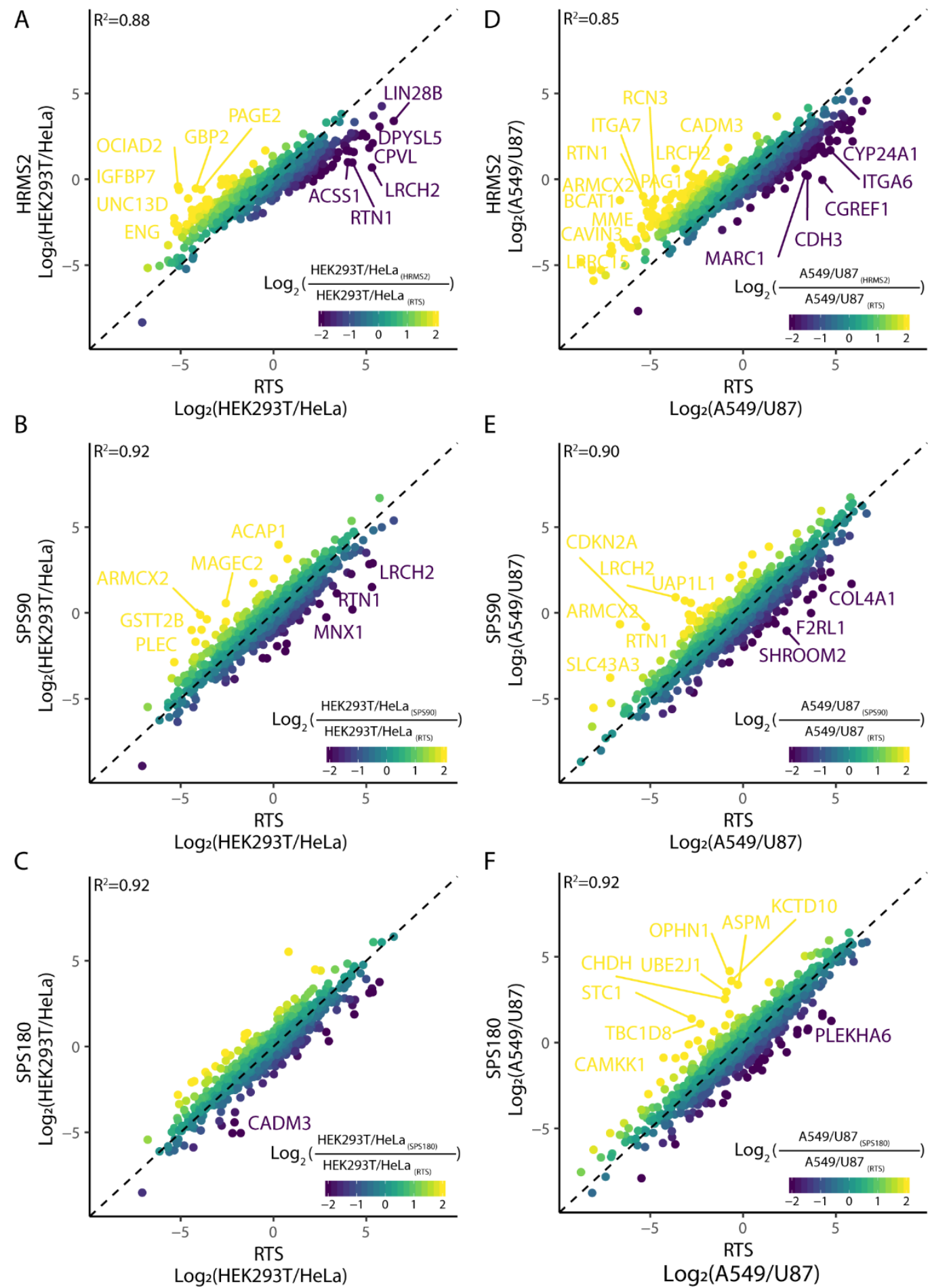

Figure S5. Quantitative comparison on additional cell lines. (A) and (D) HRMS2 vs RTS-SPS-MS3; (B) and (E) SPS90 vs RTS-SPS-MS3 and (C) and (F) SPS180 vs RTS-SPS -MS3. (A-C) Comparison of relative protein abundance between HEK293T and HeLa cell lines. (D-F) Comparison of relative protein abundance between A549 and U87 cell lines. 


\section{\# Proteins $\mathrm{w} / \mathrm{FC} \geq 2$ relative to mean in MCF7}

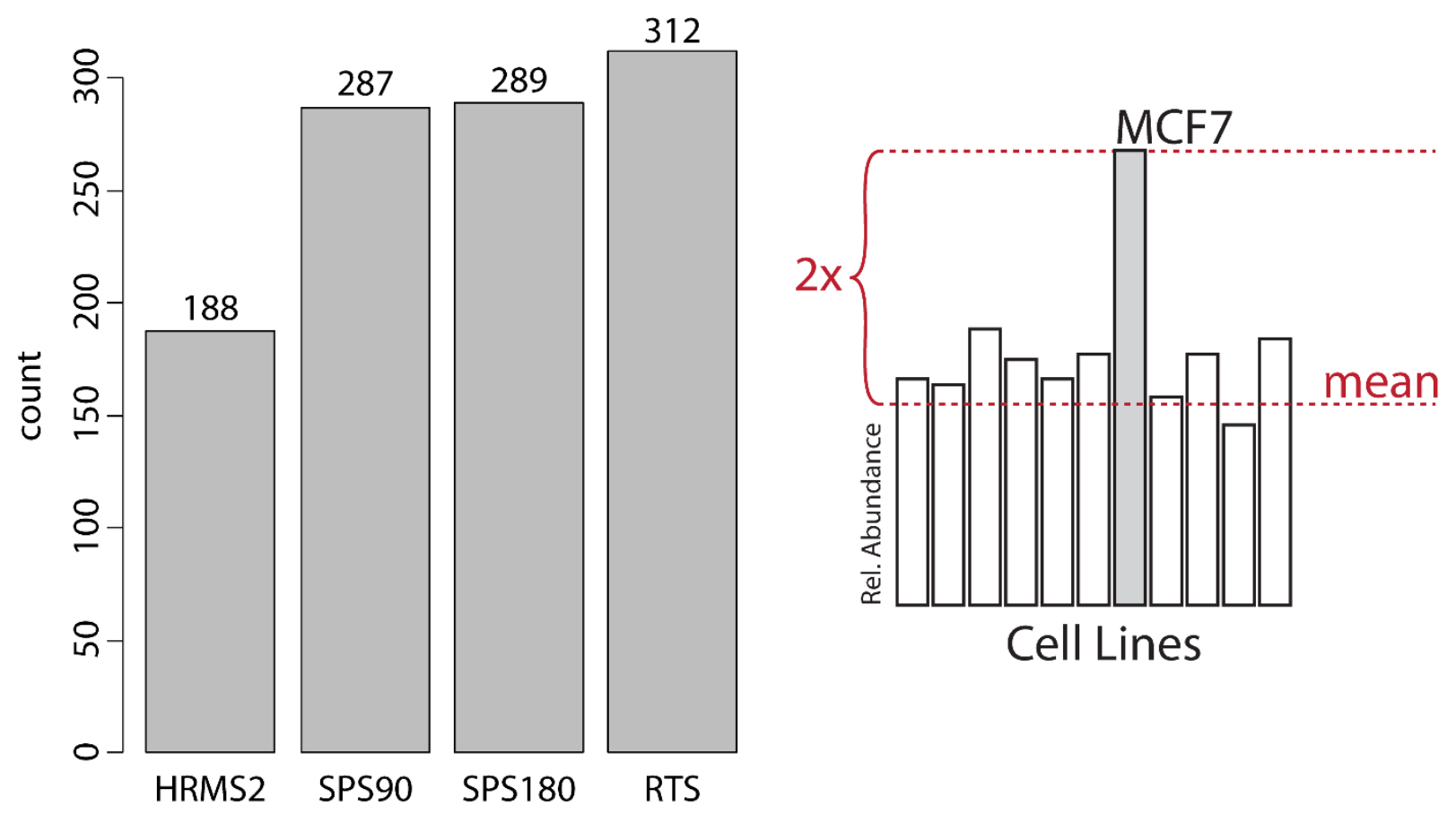

Figure S6. Total MCF7 proteins quantified with a fold change greater than two-fold compared to the mean of all cell lines. RTS-SPS-MS3 quantified 66\% more proteins with high fold changes compared to HRMS2. 


\begin{tabular}{|c|c|c|c|c|c|c|c|c|c|}
\hline & \multicolumn{4}{|c|}{ Lumos } & \multicolumn{4}{|c|}{ Eclipse } & \\
\hline \multicolumn{10}{|c|}{ Normalized isotope ratio (C13/C12) } \\
\hline Isolation & \multicolumn{9}{|c|}{$z=2$} \\
\hline Width & $\mathrm{n}$ & mean & sd & sem & $\mathrm{n}$ & mean & sd & sem & p-value \\
\hline 0.4 & 4253 & 0.0534 & 0.0285 & 0.0004 & 4859 & 0.0058 & 0.0160 & 0.0002 & $0.00 E+00$ \\
\hline 0.5 & 4250 & 0.0915 & 0.0523 & 0.0008 & 4862 & 0.0180 & 0.0215 & 0.0003 & $0.00 E+00$ \\
\hline 0.6 & 4251 & 0.1713 & 0.0950 & 0.0015 & 4861 & 0.0502 & 0.0311 & 0.0004 & $0.00 E+00$ \\
\hline 0.8 & 4252 & 0.3367 & 0.1848 & 0.0028 & 4862 & 0.1985 & 0.0509 & 0.0007 & $0.00 E+00$ \\
\hline 1 & 4250 & 0.4578 & 0.2322 & 0.0036 & 4860 & 0.5703 & 0.1055 & 0.0015 & $1.68 \mathrm{E}-173$ \\
\hline 1.2 & 4249 & 0.5708 & 0.2169 & 0.0033 & 4862 & 0.8031 & 0.0695 & 0.0010 & $0.00 \mathrm{E}+00$ \\
\hline \multirow[t]{2}{*}{2.5} & 4251 & 0.9950 & 0.0619 & 0.0009 & 4860 & 0.9963 & 0.0509 & 0.0007 & 2.62E-01 \\
\hline & \multicolumn{9}{|c|}{$z=3$} \\
\hline 0.4 & 2498 & 0.2618 & 0.1767 & 0.0035 & 2311 & 0.1441 & 0.0476 & 0.0010 & 4.66E-193 \\
\hline 0.5 & 2502 & 0.2533 & 0.1967 & 0.0039 & 2312 & 0.2690 & 0.0705 & 0.0015 & 1.97E-04 \\
\hline 0.6 & 2502 & 0.2740 & 0.1922 & 0.0038 & 2309 & 0.4635 & 0.0969 & 0.0020 & $0.00 E+00$ \\
\hline 0.8 & 2501 & 0.3783 & 0.1532 & 0.0031 & 2313 & 0.8068 & 0.1331 & 0.0028 & $0.00 \mathrm{E}+00$ \\
\hline 1 & 2501 & 0.5572 & 0.1681 & 0.0034 & 2316 & 0.9078 & 0.1040 & 0.0022 & $0.00 E+00$ \\
\hline 1.2 & 2500 & 0.7936 & 0.1387 & 0.0028 & 2316 & 0.9327 & 0.1058 & 0.0022 & 3.16E-292 \\
\hline 2.5 & 2499 & 0.9712 & 0.0898 & 0.0018 & 2312 & 0.9810 & 0.0878 & 0.0018 & 1.35E-04 \\
\hline
\end{tabular}

Table S1. Summary of normalized isotope ratio for quadrupole isolation test ( $\mathrm{z}=2$ and 3 ), related to Figure 1D. 


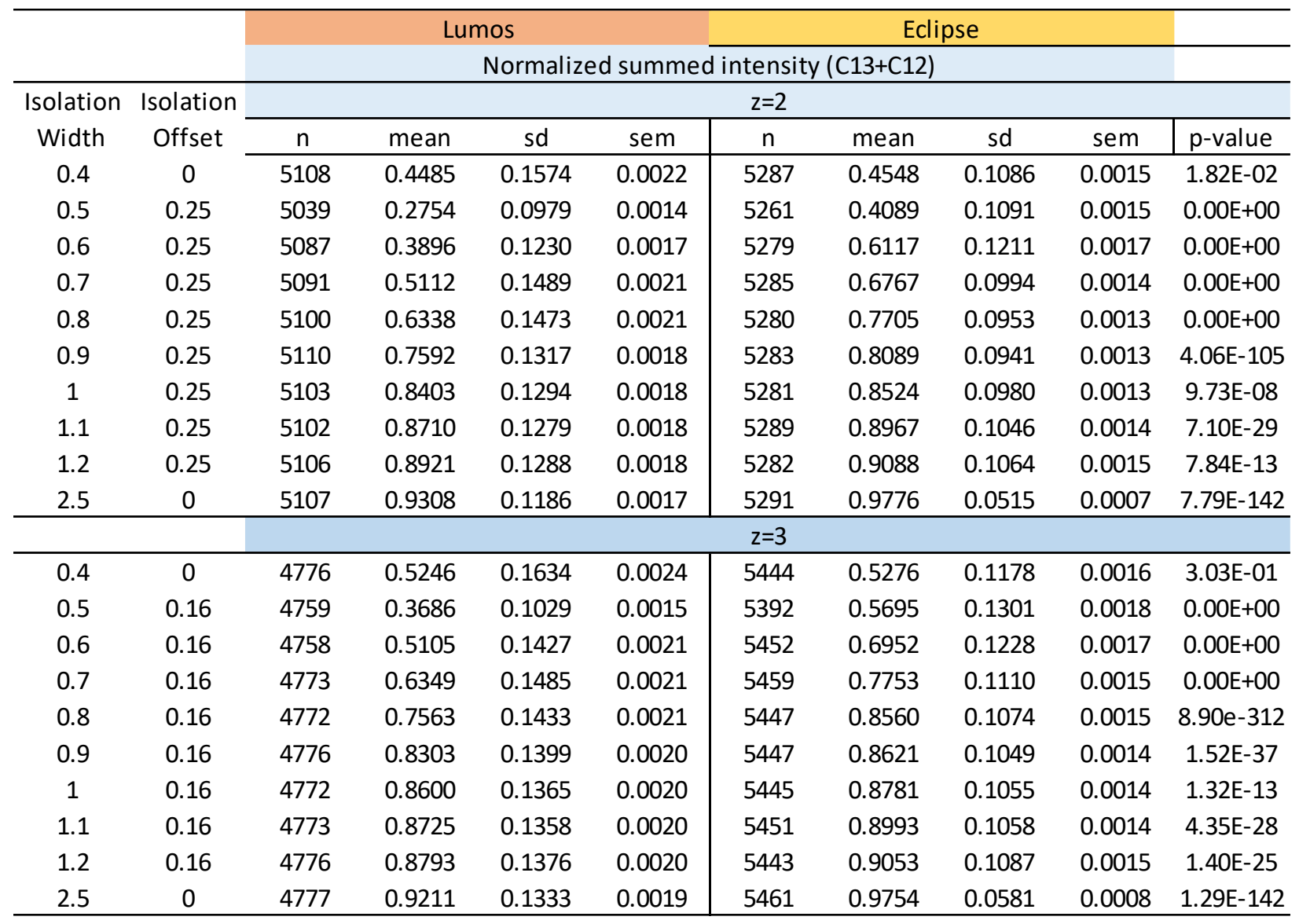

Table S2. Summary of normalized summed intensity for quadrupole isolation test with offset ( $\mathrm{z}=2$ and 3), related to Figure 1F. 